\title{
Article
}

\section{Certifier en situation d'incertitude : le cas des OGM}

\author{
Céline Granjou ${ }^{\mathrm{a}}$, Egizio Valceschini ${ }^{\mathrm{b}}$ \\ a Sociologue, CEMAGREF Grenoble, DTM, 2 rue de la Papeterie, BP 76, 38402 Saint-Martin-d'Hères cedex, France, \\ b Économiste, directeur de recherche, INRA,Unité SAD-APT, 16 rue Claude Bernard, 75231 Paris cedex, France
}

\begin{abstract}
Les consommateurs français et européens se montrent particulièrement attachés à une information claire sur les produits. En matière d'OGM, la gestion de cette information suppose une séparation sans faille entre les cultures OGM, les productions conventionnelles et biologiques. De nombreuses questions sont alors posées, tant du point de vue du cadre réglementaire, que du point de vue de l'adaptation des stratégies et des pratiques des acteurs des filières de production concernées. Dans ce cadre, on comprend que la certification se développe en soutien aux transactions marchandes. Mais comment fonctionne la certification dans un contexte réglementaire évolutif et en l'absence de connaissances stabilisées sur les preuves de l'absence d'OGM? S'inscrivant dans une approche de sociologie économique, ce texte propose une analyse originale en se focalisant sur les activités des organismes de certification.
\end{abstract}

La Rédaction

\section{Mots-clés :}

OGM; certification; industries agroalimentaires; confiance

\section{Keywords:}

GMO;

certification; food supply chain; trust
Résumé - La crédibilité de la garantie apportée par la certification provient en principe de l'expertise indépendante de l'organisme de contrôle. Dans le cas de l'information sur la caractéristique OGM ou non OGM des produits agroalimentaires, ces conditions sont insuffisantes, faute de méthodes de contrôle jugées fiables. L'article analyse le fonctionnement de la certification dans ces conditions. Il montre que son succès relatif repose, plutôt que sur une véritable clôture de la controverse sur les preuves de l' «absence » d'OGM, sur un compromis conventionnel entre les exigences des entreprises clientes et les investissements consentis par les fournisseurs. Le recours à la certification constitue un mécanisme de régulation privée considéré, au moins localement et temporairement, comme suffisant pour ne pas remettre en cause incessamment les échanges commerciaux, et en tout cas plus satisfaisant que la seule intervention des pouvoirs publics.

Abstract - Certifying in a context of uncertainty: the case of GMOs. The credibility and guarantee attached to the certification process theoretically results from the control company's independence and expertise. In the case of information on the GMO or non GMO nature of the food and agricultural products, these conditions are insufficient for lack of a stabilized definition of the control methods. However, professionals in the supply chain often turn to certification companies in order to ensure greater reliability for their exchanges concerning this information. Our paper analyses the way certification works in these situations. Certification companies are shown to use knowledge and agreements constructed by the actors concerned; they work on formalizing the good intentions of the certified industrialists and finally offer a guarantee on industrial practices based on their own reputation. Rather than supplying a definitive and universal conclusion to the controversy about proofs of GMO "absence", certification companies provide a local compromise between the demands for proof from the customer companies and the investments consented by the suppliers. Resorting to certification then constitutes sufficient proof for the industrialists, a sort of conventional agreement. Certification is a private regulating mechanism which is considered sufficient, at least locally and temporarily, not to continuously endanger the necessary coordination for market exchanges: in any case it is considered more satisfactory than the one-sided intervention of public authorities.

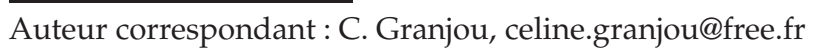




\section{Introduction}

La certification est une prestation de services réalisée par une entreprise de contrôle, rémunérée par un client (producteur ou vendeur) dans le cadre d'un contrat privé, qui se solde par l'attribution au client d'un document ayant valeur reconnue de preuve (certificat). Cette prestation a pour objectif de donner la garantie d'une instance tierce, indépendante des transactions sur un marché. Son rôle est de vérifier, au moyen d'audits et de contrôles, la conformité des pratiques ou des produits à une qualification prédéfinie, un « référentiel » dans le jargon professionnel. La certification ${ }^{1}$ produit une assurance et, par là, est censée générer de la confiance. Elle constitue un « dispositif de confiance » au sens donné à cette expression par Lucien Karpik (1996), en réalité un dispositif qui permet, en situation d'incertitude sur la qualité des produits, de stabiliser des échanges économiques durables.

Dès lors se pose la question de la crédibilité de cette garantie $^{2}$. Formellement, la crédibilité provient de l'indépendance, de l'impartialité et de la capacité d'expertise reconnues aux organismes certificateurs ${ }^{3}$ dans le cadre de procédures officielles d'accréditation. L'indépendance et l'impartialité sont censées contenir d'éventuels comportements opportunistes, l'expertise vise pour sa part à réduire les problèmes soulevés par les asymétries d'information. Il est pourtant des situations où ces supports de crédibilité sont insuffisants : lorsque la qualification du produit ou la fiabilité des méthodes de contrôle sont incertaines ${ }^{4}$.

Cette deuxième situation prévaut en matière d'information sur la caractéristique OGM (ou non $\mathrm{OGM}^{5}$ )

\footnotetext{
${ }^{1}$ La certification a été peu étudiée en tant que telle. Elle est souvent abordée dans la thématique de la qualité (Valceschini, 1995 ; Cochoy et de Terssac, 1999), notamment en lien avec un questionnement sociologique sur la normalisation industrielle (Segrestin, 1996; Gomez, 1996 ; Cochoy, 2002). Elle est aussi considérée sous un angle économique pour mettre en évidence les économies de transaction réalisées grâce au recours à des tiers (Raynaud et Sauvée, 2000), ou pour souligner la différence entre la certification de produits et la certification d'entreprise (Ravix et Romani, 1996).

2 Cette étude s'appuie sur les acquis du rapport « Pertinence et faisabilité d'une filière sans utilisation d'OGM » (Valceschini et Avelange, 2001) et constitue une partie d'une thèse de doctorat en sociologie (Granjou C., 2004. La Gestion du risque entre technique et politique. Comités d'experts et dispositifs de traçabilité à travers les exemples de la vache folle et des OGM, Université Paris 5).

3 Notés OC dans la suite du texte.

${ }^{4}$ On entend ici par qualification le fait de pouvoir attribuer une qualité et une valeur à un produit (par exemple le fait d'être, ou non, génétiquement modifié).

${ }^{5}$ Organisme génétiquement modifié. Nous utiliserons l'expression d' «absence » d'OGM ou de «non OGM » pour désigner non pas l'absence totale de traces d'ADN ou de protéines, mais l'état du produit permettant, conformément à la réglementation en vigueur et plus largement aux stratégies particulières
}

des produits agricoles et agroalimentaires, alors même qu'il s'agit d'un domaine particulièrement sensible, où les questions de sécurité sanitaire, d'information et de confiance des consommateurs sont étroitement imbriquées (Joly, 2003). Tandis que la réglementation sur l'étiquetage (Encadré) s'est efforcée de qualifier les produits OGM, demeure une incertitude juridique et technique du fait de l'incomplétude ${ }^{6}$ de l'accord entre pouvoirs publics $^{\prime}$ et acteurs professionnels sur les modalités du contrôle des OGM.

Dans ces conditions, qu'est-ce qui motive le recours des professionnels à la certification ? Comment les organismes certificateurs agissent-ils pour offrir une garantie alors que la fiabilité des méthodes de contrôle reste incertaine? Quelles fonctions assument-ils ${ }^{7}$ ?

\section{Le contrôle des OGM : une controverse sur les preuves}

\section{Les méthodes de détection : une fiabilisation insuffisante}

À partir de 1997-1998, des laboratoires privés ${ }^{8}$ sont créés, en réponse à la demande de contrôle de la présence d'OGM. Ils s'inscrivent dans le marché, en plein développement depuis les années 1990, concernant d'autres types d'analyses en agroalimentaire (caractéristiques nutritionnelles, analyses bactériologiques, etc.). Même les

des opérateurs, de ne pas étiqueter le produit comme contenant des organismes génétiquement modifiés.

${ }^{6}$ On entend ici par incomplétude le fait que les preuves justifiant l'absence d'étiquetage «OGM » des produits ne soient pas clairement définies. D’une façon générale, les termes utilisés dans ce texte renvoient à une acception large, et non pas spécifiquement juridique.

7 Pour répondre à ces questions, des monographies d'entreprises (grand semencier, petite coopérative agricole, grand amidonnier, petite entreprise de fabrication de charcuterie pâtissière, entreprise de fabrication de produits diététiques, grands distributeurs) ont été réalisées au cours d'entretiens semidirectifs avec un ou plusieurs responsables qualité des entreprises. Elles ont été complétées par des entretiens auprès des quatre principaux organismes certificateurs, auprès d'un laboratoire de détection d'OGM, ainsi que de divers acteurs institutionnels (services de la Répression des fraudes, commission de normalisation des méthodes de détection des OGM - l'entretien a été complété par l'observation participante d'une séance de la commission -, COFRAC : Comité français d'accréditation) et d'un chercheur spécialiste des méthodes de détection d'OGM.

8 Il s'agit d'anciennes sociétés prestataires d'analyses qui, après s'être lancées dans les analyses du secteur agroalimentaire depuis le milieu des années 1990, diversifient leurs activités sur un nouveau créneau (comme Wolff, Eurofins Scientific, Ampligene, filiale des laboratoires Carso...), de sociétés de biotechnologies qui se lancent dans la détection, ou encore de start-up (Calligene). On dénombre actuellement une vingtaine de laboratoires. 


\section{Encadré. Étiquetage des OGM et montée en exigence de contrôle}

La question de la qualification du contrôle de la caractéristique OGM a émergé à partir de 1996. Elle a évolué en lien étroit avec la question de la qualification des produits. Quatre temps forts rythment cette émergence.

1997-1998 : avec l'arrivée des premiers sojas américains trangéniques, une réglementation européenne d'étiquetage voit le jour. Le règlement «Novel Food » (CE 258/97) repose sur le concept d'équivalence avec l'aliment traditionnel : un produit doit être étiqueté si l'on peut y déceler, au-delà d'un seuil qui reste à définir, soit des différences nutritionnelles ou toxicologiques (problème de l'équivalence en substance), soit de l'ADN ou des protéines provenant du gène ajouté (problème de l'équivalence).

1998-1999 : les associations de consommateurs ${ }^{9}$ dénoncent les infractions à la réglementation ou ses insuffisances en arguant de tests de détection montrant que des aliments contenant des OGM sont présents sur le marché sans être étiquetés. L'association de protection de l'environnement Greenpeace élabore une «liste noire » de produits « avec OGM ». Pour être inscrits sur une « liste blanche », les fabricants doivent fournir des garanties de non-utilisation des OGM. À l'instar de la grande distribution anglaise, certains grands distributeurs français (Carrefour et Leclerc) cherchent à instaurer une traçabilité basée sur des certificats d'origine «sans OGM ». Le secteur de l'amidonnerie pèse fortement dans la diffusion de l'exigence de garantie d'absence d'OGM auprès des fournisseurs agricoles.

2000-2002 : la réglementation communautaire retient le seuil de $1 \%$ par ingrédient (règlements CE/49/2000 et CE/50/2000) comme seuil d'étiquetage OGM. Elle crée un vide juridique dans la mesure où la responsabilité d'étiquetage incombe au distributeur sans qu'aucune obligation ne soit définie concernant les fournisseurs en amont. Les distributeurs et les fabricants finaux ne laissent pas de dénoncer cette asymétrie de responsabilité. Par ailleurs, un moratoire de fait s'installe sur la culture d'OGM en Europe.

2003 : le seuil réglementaire d'étiquetage des OGM est abaissé à 0,9 \% (règlement CE/1929/2003) et l'obligation d'étiquetage est étendue à des produits qui auraient été obtenus à partir d'OGM même si aucune trace d'ADN ou de protéine n'est détectable au final. Le règlement CE 1830/2003 impose, quant à lui, l'obligation pour tout opérateur qui vend un tel produit à un autre industriel de la filière de transmettre un document mentionnant « contient des OGM » ou « produit à partir d'OGM ».

professionnels de l'agroalimentaire disposant de capacités d'expertise suffisantes pour réaliser eux-mêmes leurs analyses y ont recours. Ils ont généralement estimé qu'il était moins coûteux d'externaliser les analyses, et surtout que le recours à un tiers, évitant d'être «juge et partie", pouvait asseoir leur crédibilité.

Le marché de la détection des OGM est soumis à une concurrence très forte, marquée par une surenchère de revendication de compétence dans un contexte d'évolution permanente des techniques. Une "course » à la sensibilité des méthodes s'est engagée. Certains laboratoires dénoncent les analyses à bas prix proposées par leurs concurrents, arguant que l'ensemble de précautions et de conditions nécessaires pour fiabiliser les résultats n'est pas mis en œuvre. Un demandeur d'analyses de détection indiquait ainsi en 2000 : «Entre deux laboratoires, il y en a un qui trouvait plus de $3 \%$ d'OGM et l'autre qui ne trouvait rien!»

À partir de 1998, à la suite de la réglementation européenne d'étiquetage obligatoire des OGM, l'État a eu à assumer sa mission de contrôle de l'étiquetage concernant

\footnotetext{
960 millions de consommateurs, 316 (avril 1998); Que Choisir, 356 (janvier 1999); à nouveau 60 millions de consommateurs, 357 (janvier 2002). Contrairement aux numéros précédents, dans ce dernier cas, il ne s'agit pas de dénoncer des infractions à la loi, puisque les taux détectés sont inférieurs au seuil d'étiquetage, mais de montrer l'importance du nombre de produits contenant des traces d'OGM (environ un tiers des produits analysés).
}

les OGM ${ }^{10}$. Jusqu'en 2000, il n'y avait ni seuil au-delà duquel la présence d'OGM sans étiquetage pouvait être sanctionnée, ni analyses prouvant de façon fiable et définitive la présence ou l'absence d'OGM dans les produits $^{11}$. La tâche des agents de la Répression des fraudes consistait alors à vérifier que l'entreprise avait effectivement mis tous les moyens en œuvre pour s'assurer de l'absence d'OGM. Cette approche, fondée sur l'explicitation des formules des produits et sur la remontée des filières d'approvisionnement des entreprises enquêtées, était complétée en cas de besoin par des analyses sur produit $^{12}$.

En 2001, le nombre d'analyses effectuées par les services de la Répression des fraudes apparaît très faible (130 pour les semences et 70 pour l'alimentation humaine), comparé au nombre d'analyses effectuées en routine par un grand laboratoire privé (6000 par an), ou demandées par les professionnels (par exemple, 2500 pour un grand semencier, 75 pour une petite coopérative agricole). Les capacités de contrôle des autorités publiques n'offrent pas aux opérateurs de la filière agroalimentaire traquant

\footnotetext{
${ }_{10}$ Principalement à travers les services de la Répression des fraudes (DGCCRF), mais aussi, à un degré moindre, à travers le service de la Protection des végétaux (ministère de l'Agriculture) ainsi que les services des douanes.

11 Voir «L'étiquetage des nouveaux aliments est un leurre», La Recherche, 299, juin 1997.

12 Il s'agit de la démarche habituelle des enquêtes de la Répression des fraudes.
} 
la moindre trace d'OGM une garantie suffisante, tandis que la question se pose de savoir qui - des services publics ou des professionnels en cause - doit assumer les coûts de cette garantie.

Cependant, cette insuffisance publique n'est pas décisive, tant que l'État assure la fonction de garantie de la validité des analyses effectuées par les laboratoires privés. Il lui faut donc impérativement acquérir et conserver une compétence d'expertise publique en matière de détection d'OGM, afin de permettre à ses services de valider ou d'invalider des soupçons de contamination de semences ou de produits, et de répondre aux demandes fréquentes de professionnels confrontés à des résultats analytiques problématiques ou litigieux. Pour développer des méthodes de détection fiables, il s'appuie sur la création d'un Réseau national de détection et d'identification des $\mathrm{OGM}^{13}$.

L'Inra $^{14}$ est fortement impliqué dans ce réseau, et lui dédie une équipe de recherche. Cette équipe n'effectue ni des analyses de routine, comme le laboratoire de la Répression des fraudes, ni un travail de recherche, mais a le statut spécifique de centre d'expertise. Grâce à une connaissance pointue des conditions de réalisation des analyses, et en raison de sa capacité à négocier avec les sociétés de biotechnologie la disponibilité de matériaux de référence indispensables à la méthode $P C R^{15}$ de détection d'OGM, ce laboratoire occupe une position qui lui permet de régler, au cas par cas, de nombreux litiges ponctuels.

Reste que ce mode de régulation de la qualification OGM des produits n'offre pas de solution globale au problème de la forte variabilité des résultats analytiques ni à celui des surenchères de revendication de compétences de la part des laboratoires. La définition de méthodes normalisées ${ }^{16}$ apparaît indispensable pour créer les conditions d'une stabilisation des relations entre opérateurs et laboratoires, ainsi qu'entre clients et fournisseurs industriels. Cette définition permettra l'aboutissement d'un

\footnotetext{
${ }^{13}$ Composé du laboratoire de la DGCCRF de Strasbourg, du GEVES (Groupe d'étude et de contrôle de la variété et des semences, regroupant des chercheurs de l'Inra et des professionnels) et d'une équipe Inra de Versailles.

${ }^{14}$ Institut national de la recherche agronomique.

15 Polymerase Chain Reaction : cette réaction nécessite de disposer d'une séquence d'ADN caractéristique du gène introduit par transgenèse; elle consiste alors à détecter cette séquence dans le produit analysé, puis à la quantifier (grâce à des techniques de purification et de multiplication de l'ADN).

${ }_{16}$ Une norme est « une spécification technique ou un autre document accessible au public, établi avec la coopération et le consensus ou l'approbation générale de toutes les parties intéressées, fondé sur les résultats conjugués de la science, de la technologie et de l'expérience, visant à l'avantage optimal de la communauté dans son ensemble et approuvé par un organisme qualifié » (Igalens et Penan, 1994).
}

projet d'accréditation officielle ${ }^{17}$ des laboratoires, dont l'objectif est de garantir, grâce à un logo apposé sur les bulletins d'analyses, que le laboratoire a suivi une procédure analytique éprouvée.

\section{L'arène de la normalisation : un apprentissage collectif limité}

Les négociations sur la normalisation de méthodes de détection d'OGM sont menées au sein d'une arène orchestrée par la commission Afnor ${ }^{18}$ créée en 1998. Elles confrontent les laboratoires d'analyse, privés et publics, la Répression des fraudes, les professionnels (semenciers, amidonniers, Fédération du commerce et de la distribution...), les fabricants d'appareils de détection, les associations de consommateurs. Elles s'appuient sur l'expérience des laboratoires ainsi que sur la réalisation de ring-tests, ou tests inter-laboratoires, visant à accumuler des données sur les caractéristiques statistiques des techniques utilisées (linéarité, spécificité, répétabilité - dans un même laboratoire-, reproductibilité-d'un laboratoire à un autre -, marges d'incertitude obtenues en fonction du pourcentage trouvé).

L'objectif est la définition des conditions d'une «bonne analyse » (matériel et formation nécessaires, précautions à respecter, etc.). Au regard du marché du contrôle, l'enjeu est d'éviter les conduites publicitaires opportunistes $^{19}$ de certains laboratoires qui arguent d'une sensibilité de détection extrêmement puissante, alors même que les résultats trouvés ne sont pas reproductibles, ou supposent le prélèvement de masses commercialement aberrantes du produit.

Dans cette arène, les professionnels peuvent comparer et juger par eux-mêmes de la fiabilité des différentes pratiques et méthodes d'analyse. Entre eux, les laboratoires échangent certaines connaissances et améliorent leurs compétences respectives. Confrontés aux professionnels, ils prennent mieux la mesure de la problématique du contrôle des OGM en milieu industriel, notamment les difficultés liées à l'échantillonnage et la nécessité d'aboutir à des résultats statistiquement reproductibles.

La commission Afnor publie en décembre 2000 une norme expérimentale, ou guide de «bonnes pratiques générales ». Elle est utilisée par les professionnels et par les organismes certificateurs eux-mêmes pour sélectionner les laboratoires d'analyse. Toutefois, rien, actuellement, ne garantit le respect de ces bonnes pratiques par les laboratoires. Les normes définitives, qui seules pourront

\footnotetext{
17 L'accréditation est donnée par le COFRAC pour une méthode d'analyse, à partir d'un guide d'audit décrivant des exigences techniques propres à cette méthode ainsi que les exigences d'assurance qualité assurant les bonnes pratiques du laboratoire (norme 17 025).

18 Association française de normalisation.

19 Au sens donné à ce terme en économie.
} 
donner lieu à une accréditation par le COFRAC des laboratoires les respectant, ne sont prévues que pour $2005 \mathrm{ou}$ $2006^{20}$. Les preuves du contrôle des OGM restent donc insuffisantes.

Sur le plan technique, la fiabilisation des preuves analytiques se heurte par ailleurs à des décalages entre les capacités techniques et les exigences de la réglementation. Cette dernière mentionne un seuil absolu, alors que les analyses ne peuvent fournir qu'un pourcentage pourvu $d^{\prime}$ 'une marge d'incertitude ${ }^{21}$.

Par ailleurs, au sein de la commission, le climat est à la concurrence entre les divers professionnels, tandis que les consommateurs sont finalement très peu présent $^{22}$. Entre laboratoires, l'échange d'informations demeure difficile compte tenu du fait qu'il s'agit de dévoiler aux concurrents ses propres méthodes mises au point en interne. Certains industriels essaient $d$ 'imposer leurs intérêts, en revendiquant, par exemple, une élévation du seuil de détection analytique qui leur permettrait de limiter les exigences imposées aux filières non OGM. Les sociétés de biotechnologies produisant des standards de référence indispensables pour détecter chaque type d'OGM et pour "caler» les méthodes analytiques rechignent très souvent à fournir ces matériaux, malgré une réglementation devenue plus contraignante depuis fin 2002.

La controverse sur la suffisance des preuves et des contrôles fondant la qualification OGM des produits est donc loin d'être close, malgré une meilleure connaissance partagée des pratiques de détection.

Les grands $\mathrm{OC}^{23}$ présents sur le marché ne s'y trompent pas. Il est intéressant de noter que, parmi les plus importants, seuls SGS et BVQI sont entrés dans la démarche d'attestation concernant les OGM, et encore, semble-t-il, avec quelques hésitations en ce qui concerne la SGS. A contrario, l'AFAQ estime que le cas des OGM est trop épineux pour qu'ils engagent leur responsabilité dans une attestation, au point d'avoir refusé de répondre à des demandes de clients dans ce sens. Cette stratégie correspond à une image "anti-marketing ", liée à une

\footnotetext{
${ }^{20}$ Il existe toutefois une possibilité d'accréditation plus restreinte, dite « hors programme ", mise en œuvre dès à présent par certains laboratoires.

${ }^{21}$ Surtout, la réglementation impose un pourcentage en masse, alors que les techniques PCR détectent des gènes et donnent des résultats en pourcentage de molécules, qu'il n'est pas évident de traduire en pourcentage de masse. Le pourcentage en gènes surestime le pourcentage en masse, du fait de l'existence possible d'un ou deux gènes pour une molécule d'ADN.

${ }^{22}$ Une seule personne représente les associations de consommateurs, et elle n'est pas toujours présente.

23 AFAQ (Association française pour l'assurance de la qualité), SGS (Société générale de surveillance) et BVQI (Bureau Veritas Quality International).
}

réputation de sévérité, entretenues par l'AFAQ ${ }^{24}$. Pour eux, le problème de la fiabilité analytique est important, et le marché de la certification concernant les OGM est jugé peu sérieux. Le responsable AFAQ commente ainsi le choix des organismes certificateurs concurrents : «Ils considèrent que les progrès technologiques actuels ne peuvent pas certifier à $100 \%$ le respect de la réglementation, les $1 \%$ [...]. J'en ai discuté il y a quelque temps avec eux et ils me disaient bien qu'à chaque fois où ils s'engageaient en signant le certificat, ils n'étaient pas toujours très à l'aise dans leurs baskets [...]. »

Comment les organismes certificateurs concernés s'accommodent-ils alors de ces incertitudes techniques et juridiques sur les preuves de l'absence d'OGM, et parviennent-ils à répondre à la demande des industriels clients?

\section{L'organisme certificateur : une régulation privée pour une qualification non stabilisée}

\section{Les logiques de recours à la certification : réputation et garantie durable}

Dans cette situation d'incertitude, nombre de professionnels font appel à des organismes de certification pour fiabiliser les échanges concernant la qualification OGM des produits (Valceschini et Avelange, 2001); ce recours peut répondre à des objectifs différents.

Certaines entreprises recherchent la caution d'une tierce partie engageant sa propre responsabilité dans les attestations, et fournissant une garantie jugée supérieure à celle de l'entreprise. Il s'agit d'avoir une caution de la part d'une autorité dont la caractéristique est finalement moins d'être tierce, impartiale, que d'être perçue comme supérieure du fait d'un risque de réputation plus élevé. Les petites et moyennes entreprises soumises à la logique des marques de distributeurs, dont l'engagement n'a pas la crédibilité de celui d'une multinationale, sont ici les plus concernées. Un responsable d'organisme certificateur indique : «C'est la caution de l'organisme certificateur, parce que bon, le producteur, mettons Nestlé: Nestlé s'engage en disant "nous, nous n'avons pas d'OGM dans nos produits". C'est bien, Nestlé, on peut peut-être les croire, maintenant le producteur Lambda, la charcuterie, je ne sais qui au fin fond du Larzac s'engageant sur une telle mention : la caution n'est pas la même. »

D'autres entreprises, de plus grande taille, recourent également au service d'un OC, moins par recherche d'une autorité supérieure que dans un objectif de délégation

\footnotetext{
${ }^{24}$ Cette attitude correspond également au fait que la vocation première de l'AFAQ est la certification de système, alors que la certification de produit n'est entrée que plus tard dans les compétences de l'association.
} 
d'une charge de travail. Pour les entreprises d'amidonnerie, par exemple, le recours à une démarche de certification de leurs propres pratiques permet de substituer un audit unique à une multiplicité d'audits effectués par leurs clients et les clients de leurs clients.

Toutefois, certains grands fabricants et distributeurs gardent une préférence marquée pour le contrôle des produits reposant directement sur l'analyse. Ils effectuent leurs propres audits chez leurs fournisseurs, collectant les engagements signés des fournisseurs de leurs fournisseurs et les résultats des analyses effectuées, avant d'en réaliser eux-mêmes. Selon eux, la certification serait surtout une prestation de service soumise à un phénomène de «business » de la part des OC soucieux de développer leur marché. Elle resterait incapable d'apporter une preuve aussi définitive que le bulletin d'analyses. Un semencier déclare ainsi :

«Ils peuvent certifier que vous êtes dans une filière non OGM, mais ils ne certifient pas le produit. C'est une obligation de moyen, mais pas de résultat : ils peuvent dire effectivement que l'entreprise met en place tous les process, tous les contrôles nécessaires sur la base d'un référentiel interne et certifier sur ce document; mais ce n'est pas un référentiel qui est normalisé, c'est un référentiel interne à l'entreprise qui est écrit par le personnel de l'entreprise! [...] Moi, si je suis un industriel en bout de chaîne, je préfère que vous n'ayez rien de tout ça et que vous contrôliez les produits que vous commercialisez! »

Au final, si la garantie de la certification n'est pas nécessairement parfaite aux yeux des entreprises clientes, l'intervention d'un organisme certificateur stabilise dans certains cas un compromis entre le degré de caution exigé par le client et les investissements possibles ou consentis par le fournisseur. Elle permet à tout le moins d'obtenir une garantie sur la maîtrise des processus, jugée plus solide parce que pérenne, permanente, alors que les analyses ne fourniront jamais que des vérifications ponctuelles, qu'il faudrait idéalement renouveler sur chaque lot et chaque produit. La logique de certification implique une garantie durable dans le temps : le certificat est renouvelé au moyen d'audits répétés, tandis que l'entreprise tient l'OC au courant de certaines évolutions de sa situation. A contrario, lorsque le demandeur (généralement un grand distributeur) rémunérant l'organisme certificateur cherche simplement une garantie ponctuelle concernant les pratiques d'un de ses fournisseurs, il obtient un rapport sur le fournisseur, qui atteste de la « maîtrise du risque OGM » ou de sa non-maîtrise, mais cette expertise est ponctuelle.

Pour autant, le service d'un organisme certificateur n'est pas ici une certification au sens propre du terme, dans la mesure où le cahier des charges n'est pas garanti par une instance indépendante telle que la Commission nationale des labels et de la certification (CNLC), dans le cas des signes officiels de qualité. Il s'agit d'une attestation qui entre dans la catégorie des contrôles privés, basés sur des référentiels élaborés par les entreprises ou les OC, ou les deux.

La spécificité du contrat de contrôle privé tient ainsi au fait que des obligations contractuelles lient l'organisme certificateur et le fournisseur, alors que le bénéficiaire effectif est le client du fournisseur. L'autorité publique (la Répression des fraudes) peut contrôler qu'il n'y a pas utilisation abusive du nom d'un organisme certificateur par un fournisseur en vérifiant la présence effective d'un contrat entre l'entreprise et l'organisme. En revanche, les termes mêmes du contrat sont laissés au seul libre arbitre des parties prenantes, à la différence des démarches que supervise, par exemple, la CNLC.

Comment le travail effectif des organismes certificateurs peut-il alors assurer la crédibilité d'un tel mécanisme du point de vue des entreprises soucieuses de s'assurer de la qualité des produits qu'elles achètent? L'attestation délivrée ne tient guère d'un « dispositif de jugement $t^{25}$ ", dans la mesure où elle fournit peu d'informations sur l'entreprise auditée. Pour réduire le soupçon selon lequel l'industriel comme le certificateur pourraient avoir intérêt à maintenir leur contrat en étant peu exigeants vis-à-vis des critères de certification, les OC développent une accumulation d'indices d'autorité ainsi qu'une rhétorique nuancée définissant leur propre apport. Leur activité repose ici sur un engagement de réputation, sur la capitalisation de diverses sources d'expertise et de connaissances, ainsi que sur la formalisation des bonnes intentions de l'industriel certifié.

\section{Les fondements de l'attestation : des «bonnes pratiques » aux « bonnes intentions »}

Le principe de l'attestation fournie par l'OC sur les OGM consiste moins à contrôler le produit sortant de l'entreprise, qu'à vérifier que l'entreprise sait vérifier, non pas que le produit est conforme, mais qu'elle a évité un certain nombre de situations de non-conformité. Les termes des documents délivrés, par exemple par Bureau Veritas ou par la SGS, désignent respectivement leur apport comme la «reconnaissance » et l'«évaluation » du «système de maîtrise des approvisionnements et de la préservation d'identité » des céréales transformées par les usines, lesquels systèmes sont définis comme « visant à garantir la livraison de produits finis élaborés à partir de maïs non OGM $(<0,1 \%) \ldots »^{26}$.

\footnotetext{
${ }^{25}$ Karpik (1996) qualifie un dispositif de confiance de «dispositif de jugement", dans la mesure où il y a une mise en visibilité de certaines pratiques de l'entreprise.

${ }^{26}$ Les citations sont extraites de documents d'attestations délivrés par BV et la SGS aux entreprises auditées pour l'année 2002, et dont les enquêtés nous ont donné une copie. Le seuil de $0,1 \%$ correspond à une exigence des acteurs de l'amidonnerie, et n'a jamais eu d'existence réglementaire.
} 
Le référentiel définissant les audits à effectuer est élaboré en interne, par l'organisme certificateur, mais peut être adapté aux contraintes économiques des industriels, notamment en ce qui concerne la composition des céréales utilisées, indiquée sur le document d'attestation $(0,1 \%$ généralement pour l'alimentation humaine, $1 \%$ pour l'alimentation animale). Les grandes sections du référentiel élaboré par Bureau Veritas sont focalisées sur la maîtrise par l'entreprise des possibilités d'entrée des OGM dans l'usine lors des approvisionnements, puis des possibilités de contamination lors des opérations de stockage, transport et transformation, et enfin sur ses aptitudes à reconstituer la provenance et le devenir des lots de produits. La conformité des entreprises au référentiel est vérifiée lors d'audits annuels ou parfois semestriels. La fréquence et le caractère prévisible des audits ne permettent cependant pas de contrôler les pratiques routinières. Les audits reposent sur des preuves indirectes et ad hoc, telles que les documents conservés par l'entreprise et la réussite d'exercices de traçabilité.

L'apport de l'OC réside donc dans la formalisation de critères de bonnes pratiques (comme la définition de la quantité de produit déclassé lors du nettoyage d'un appareil ou la fréquence des analyses de vérification), alors que le contrôle même de ces pratiques reste indirect. Le travail des OC permet de repérer et de formaliser les moyens génériques à disposition des entreprises dans l'objectif de fournir un certain type de produits. Il s'agit, selon les termes d'un responsable d'OC, que «l'entreprise ait pris conscience et ait réussi à évaluer son risque ». S'il ne s'agit pas de garantir la qualité mesurable des produits, il ne s'agit pas non plus de contrôler directement les bonnes pratiques des entreprises, mais plutôt de formaliser et de cautionner des «bonnes intentions ». Comme le suggèrent les expressions employées par les responsables d'OC, qui déclarent contrôler la "prise de conscience » ou encore la «politique » de l'entreprise, il s'agit de garantir en fin de compte l'engagement de l'entreprise, sa volonté de fournir des produits «non OGM».

Dans le même sens, les organismes certificateurs ne font pas effectuer d'analyses régulières, mais jugent plutôt de la fiabilité des laboratoires de détection retenus par les entreprises. Le souci de qualification des laboratoires est ici nettement plus important que pour une certification de produit classique. Face à la carence de procédures codifiées de reconnaissance des laboratoires, les OC s'emploient à sélectionner - « référencer », selon leurs termes des laboratoires jugés dignes de foi, au moyen d'un montage de divers signes de reconnaissance. Ils empruntent à diverses sources d'expertise susceptibles de faire autorité, alliant leur propre expertise à l'expertise développée par d'autres acteurs.

L'OC ne peut juger de la fiabilité du laboratoire que par l'intermédiaire de signes d'autorité très indirects. Sont ainsi cités par les responsables certificateurs le fait que le laboratoire soit accrédité pour d'autres techniques que celles de détection des OGM, sa possession d'accréditations étrangères, son ancienneté même, ainsi que, selon une sorte de processus tautologique, le fait que l'entreprise cliente, disposant elle-même d'un niveau d'expertise élevé, ait procédé à une sélection rigoureuse sur le fondement d'audits et de tests. La caution de l'OC quant à la fiabilité du laboratoire utilisé renvoie finalement à une confiance dans le jugement initial de l'entreprise certifiée.

Pour les démarches de filière, quand l'objectif est une attestation pour allégation à destination du consommateur final, l'OC est davantage engagé du fait de l'apposition de son nom sur l'allégation finale portée sur l'emballage du produit. Il définit lui-même, dans ce cas, le plan de contrôle analytique, qui implique le choix du laboratoire ainsi que de la méthode d'échantillonnage. Comme l'OC commande lui-même les analyses et rémunère le laboratoire, il peut procéder à de véritables audits du laboratoire, et vérifier, par exemple, que le laboratoire applique bien la norme Afnor expérimentale. Le niveau d'expertise propre de l'OC doit alors être plus élevé, tandis que dans le cas d'une démarche d'entreprise, des sources externes de reconnaissance du laboratoire (référencement par un client important, ancienneté du laboratoire) seront davantage mobilisées.

\section{Régulation de la qualification : le marché plutôt que l'État?}

La démarche d'attestation liée aux OGM s'inscrit dans un mouvement général de multiplication des cahiers des charges et notamment des référentiels de contrôle privés. Ceux-ci répondent à une exigence croissante de connaissance des étapes et des conditions de production, au détriment des caractéristiques du produit final ${ }^{27}$. Ils constituent une régulation alternative des échanges entre entreprises, dans des situations où l'expertise et le contrôle des pouvoirs publics sont limités.

Un professionnel, commentant l'évolution des exigences des clients vis-à-vis de la caractéristique OGM des produits, indique cette coexistence de deux systèmes de régulation de la loyauté des échanges entre professionnels : selon que les produits fabriqués sont destinés à un grand distributeur ou à un grossiste, sera mobilisé le seul système de régulation classique, reposant sur la garantie publique de la loyauté des échanges, ou le système reposant sur une accumulation de documents et d'analyses cautionnée par un organisme tiers.

La crédibilité du fabricant renvoie dans un cas à un système juridique global selon lequel la Répression des

\footnotetext{
27 Dans le cas des plans de contrôle de sécurité sanitaire, qui prolifèrent tout particulièrement aux dires des professionnels, les démarches passent souvent par une connaissance en amont des sources possibles de contamination dans l'environnement général de fabrication des produits.
} 
fraudes peut sanctionner toute allégation mensongère entre opérateurs, et, dans l'autre, exige une construction de preuves explicites et l'intervention de la caution d'un OC. Ce niveau d'exigence croissant quant aux assertions des professionnels sur les marchés intermédiaires ressort également de l'émergence de demandes de preuves concernant des substances déjà interdites par la réglementation : " $C^{\prime}$ est réglementaire : forcément, on ne va pas le faire! Vous voyez, c'est ça qui est agaçant. On est tenu de respecter la réglementation, donc ça devrait suffire! Eh bien non, il faut quand même que vous attestiez qu'effectivement on ne le fait pas... » (un professionnel).

Cette évolution de la régulation des échanges intermédiaires entre entreprises se comprend dans la perspective des manquements que l'on vise à éviter. Si le système traditionnel de contrôle est conçu pour rechercher les fraudes, les infractions volontaires, actuellement, il s'agit plutôt d'exclure les négligences, l'insuffisance de précautions et de mesures. Dans ce cadre, attestation, certification et accréditation sont particulièrement utilisées afin de fiabiliser les échanges, alors même que tous les acteurs reconnaissent simultanément leur caractère conventionnel : les industriels interviewés précisent ainsi unanimement que, si l'accréditation est un critère primordial dans leur choix d'un laboratoire d'analyse, cette accréditation ne garantit pas la justesse à proprement parler des résultats analytiques, mais plutôt le fait qu'on ne pourra rien leur reprocher dans la mesure où ils auront fait appel à un laboratoire accrédité.

\section{Conclusion}

La certification en matière d'OGM cherche à répondre au problème crucial de la confiance des consommateurs dans l'information sur la présence/absence d'OGM dans les produits et dans les processus de production. Ce problème engendre une exigence de transparence accrue dans les échanges intermédiaires entre entreprises, alors que la qualification des produits du point de vue de la caractéristique OGM a lieu dans un univers de connaissances controversées, traduit dans l'univers de l'action économique par des règles non stabilisées. Incomplétude de la réglementation quant à la définition des obligations mutuelles et des responsabilités exactes des acteurs, fiabilité limitée des résultats analytiques et capacités de détection insuffisantes pour fournir une preuve reconnue de présence/absence d'OGM, harmonisation limitée des techniques de contrôle et de mesure concourent à créer une situation d'insécurité juridique, alors même que les professionnels des filières agroalimentaires «découvrent» la complexité de leurs circuits $\mathrm{d}^{\prime}$ approvisionnement $\mathrm{t}^{28}$.

\footnotetext{
${ }^{28}$ La mise en place de procédures de traçabilité, visant une
} transparence accrue par rapport à cette complexité des circuits
Le cas des OGM comme celui, souvent évoqué par ailleurs, des allergène ${ }^{29}$ montrent que ces nouvelles exigences de transparence en situation d'incertitude tendent à être investies par les professionnels selon des formes de régulation privée. L'extension des informations jugées nécessaires demande en effet des capacités d'expertise de plus en plus poussées qui induisent l'utilisation de techniques controversées. Elles supposent la maîtrise de sources possibles de contamination extrêmement variées et parfois peu prévisibles, touchant aux ingrédients les plus insignifiants ainsi qu'à des processus de fabrication dont les résultats ne sont, parfois, tout simplement pas détectables au final. Les professionnels des filières agroalimentaires font alors appel à des organismes de certification pour fiabiliser leurs échanges concernant la qualification OGM des produits. Ces tierces parties s'insèrent dans les relations fournisseur-client, sélectionnent les laboratoires d'analyses et supervisent les professionnels. Tandis que les divers acteurs sont engagés dans des constructions d'accords sur la qualification «OGM » des produits, impliquant notamment une normalisation des méthodes d'analyse au sein de l'Afnor, les OC capitalisent ces accords et ces connaissances, et promettent, en engageant leur réputation, une garantie permanente et éclairée sur les pratiques professionnelles.

Plutôt qu'une clôture définitive et universelle de la controverse sur les preuves de $l^{\prime}$ «absence » d'OGM lors des échanges à l'intérieur des filières, le succès de l'intervention des organismes certificateurs apparaît finalement comme un compromis local entre les demandes de preuves de la part des entreprises clientes et les investissements consentis par les fournisseurs. Le recours à la certification fonde une forme d'accord conventionnel fonctionnant comme preuve suffisante ; cet accord repose sur une échelle de réputation des entreprises - la caution de l'OC étant généralement jugée supérieure à celle de l'entreprise certifiée, mais pouvant aussi, dans certains cas, prendre appui sur elle - ainsi que sur un travail de formalisation des bonnes intentions des industriels certifiés.

La caution que représentent les OC fournit alors à certaines entreprises de l'agroalimentaire, confrontées aux exigences croissantes de transparence dans un contexte de problèmes de sécurité sanitaire, un mécanisme privé jugé, au moins localement et temporairement, suffisant pour ne pas remettre en cause incessamment la

de production, rencontre des problèmes très semblables à ceux que pose la certification (Boy, 2003).

29 Soulignons que les allergènes présentent le même type de problème que les OGM du point de vue des échanges. Les obligations des opérateurs sont peu cadrées par la réglementation, et les méthodes analytiques sont insuffisantes pour trancher les litiges entre acteurs, du fait notamment de la diversité des sources possibles de contamination au court de la circulation et de la fabrication des produits. 
coordination nécessaire aux échanges commerciaux. Le recours à la certification constitue une régulation privée qui, sans susciter l'unanimité sur sa valeur, est jugée suffisante par certains opérateurs, et en tout cas plus satisfaisante que la seule implication des pouvoirs publics.

\section{Références}

Boy, L., 2003. Union européenne, précaution et traçabilité des OGM, in Pedrot, P., Traçabilité et responsabilité, Paris, Economica, 240-356.

Cochoy, F., 2002. Une petite histoire du client, ou la progressive normalisation du marché et de l'organisation, sociologie du travail, 44, 3, 357-380.

Cochoy, F., de Terssac, G., 1999. Les enjeux organisationnels de la qualité : une mise en perspective, Sciences de la société, 46, 3-18.

Gomez, P.-Y., 1996. Normalisation et gestion de la firme : une approche conventionnaliste, Revue d'économie industrielle, 75, 113-131.

Granjou, C., 2004. La Gestion du risque entre technique et politique. Comités d'experts et dispositifs de traçabilité à travers les

Reçu le 20 novembre 2003. Accepté le 2 septembre 2004. exemples de la vache folle et des OGM. Thèse de doctorat en sociologie, Université Paris 5.

Igalens, J., Penan, H., 1994. La Normalisation, Paris, PUF.

Joly, P.-B., 2003. Les OGM en débat. Vers de nouveaux modes de gouvernance de l'innovation et des risques, OCL, 10,3 .

Karpik, L., 1996. Dispositifs de confiance et engagements crédibles, Sociologie du travail, 4, 527-550.

Ravix, J.T., Romani, P.M., 1996. Certification et formes de coordination dans l'organisation de la production industrielle, Revue d'économie industrielle, 75, 275-290.

Raynaud, E., Sauvée, L., 2000. Signes collectifs de qualité et structures de gouvernance, Économie rurale, 258, 101-111.

Segrestin, D., 1996. La normalisation de la qualité et l'évolution de la relation de production, Revue d'économie industrielle, 75, 291-307.

Valceschini, E., 1995. Entreprises et pouvoirs publics face à la qualité, in Allaire, G., Boyer, R. (Eds), La Grande Transformation de l'agriculture, Paris, Economica.

Valceschini, E., Avelange, I., 2001. Analyse économique et réglementaire de l'organisation d'une filière «sans OGM ». Rapport du programme «Pertinence économique et faisabilité d'une filière sans utilisation d'OGM », Paris, Inra. 